\title{
Optimization of process performance by multiple pentagon fuzzy responses: Case studies of wire-electrical discharge machining and sputtering process
}

\author{
Al-Refaie, A. ${ }^{a}$, Lepkova, N. ${ }^{b,}{ }^{,}$, Abbasi, G. ${ }^{a}$, Bani Domi, G. ${ }^{a}$ \\ aniversity of Jordan, Department of Industrial Engineering, Jordan \\ ${ }^{b}$ Vilnius Gediminas Technical University, Department of Construction Management and Real Estate, Lithuania
}

\begin{abstract}
A B S T R A C T
This research developed mathematical models to optimize process performance for multiple pentagon fuzzy quality responses. Initially, each quality response was represented by a pentagon membership function. Then, the combination of optimal factor levels was obtained for each response replicate. Those optimal combinations were then used to construct pentagon regression models for each response. A pentagon fuzzy optimization model was formulated and solved to determine the combination of optimal factor levels at each element of pentagon response's fuzzy number. Two real case studies, i.e. wireelectrical discharge machining and sputtering process, were provided for illustration. Optimal results of the two case studies revealed that the proposed procedure effectively optimized performance under uncertainty and provided larger improvement in multiple quality characteristics. In conclusion, the proposed procedure may enhance the process engineer's knowledge about effects of uncertainty on process/product performance and help practitioners decide the proper adjustments of factor levels in order to enhance performance of electrical discharge machining and sputtering process.
\end{abstract}

(c) 2020 CPE, University of Maribor. All rights reserved.

\author{
ARTICLE INFO \\ Keywords: \\ Modeling and optimization; \\ Fuzzy goal programming; \\ Pentagon regression modelling; \\ Pentagon fuzzy numbers; \\ Wire electro-discharge machining \\ (WEDM); \\ Surface roughness (SR); \\ Material removal rate (MRR); \\ Sputtering process; \\ Gallium-doped ZnO (GZO) \\ *Corresponding author: \\ natalija.lepkova@vgtu.lt \\ (Lepkova, N.) \\ Article history: \\ Received 7 August 2020 \\ Revised 16 October 2020 \\ Accepted 19 October 2020
}

\section{References}

[1] Athreya, S., Venkatesh, Y.D. (2012). Application of Taguchi method for optimization of process parameters in improving the surface roughness of lathe facing operation, International Refereed Journal of Engineering and Science, Vol. 1, No. 3, 13-19.

[2] Liao, H.-C., Chen, Y.-K. (2002). Optimizing multi-response problem in the Taguchi method by DEA based ranking method, International Journal of Quality \& Reliability Management, Vol. 19, No. 7, 825-837, doi: 10.1108/ $\underline{02656710210434766}$

[3] Lin, C.L. (2004). Use of the Taguchi method and grey relational analysis to optimize turning operations with multiple performance characteristics, Materials and Manufacturing Processes, Vol. 19, No. 2, 209-220, doi: 10.1081/AMP-120029852.

[4] Hocine, A., Kouaissah, N., Bettahar, S., Benbouziane, M. (2018). Optimizing renewable energy portfolios under uncertainty: A multi-segment fuzzy goal programming approach, Renewable Energy, Vol. 129, Part A, 540-552, doi: 10.1016/j.renene.2018.06.013.

[5] Komsiyah, S.; Meiliana; Centika, H.E. (2018). A fuzzy goal programming model for production planning in furniture company, Procedia Computer Science, Vol. 135, 544-552, doi: 10.1016/i.procs.2018.08.207.

[6] Mirzaee, H., Naderi, B., Pasandideh, S.H.R. (2018). A preemptive fuzzy goal programming model for generalized supplier selection and order allocation with incremental discount, Computers \& Industrial Engineering, Vol. 122, 292-302, doi: 10.1016/i.cie.2018.05.042. 
[7] Johnson, D.B., Bogle, I.D.L. (2019). A quantitative risk analysis approach to a process sequence under uncertainty - A case study, Computers \& Chemical Engineering, Vol. 126, 1-21, doi: 10.1016/i.compchemeng.2019.03.039.

[8] Al-Refaie, A., Musallam, A. (2018). Using mixed goal programming to optimize performance of extrusion process for multiple responses of irrigation pipes, Proceedings of the Institution of Mechanical Engineers, Part E: Journal of Process Mechanical Engineering, Vol. 233, No. 2, 412-424, doi: 10.1177/0954408918781624.

[9] Yaghoobi, M.A., Jones, D.F., Tamiz, M. (2008). Weighted additive models for solving fuzzy goal programming problems, Asia-Pacific Journal of Operational Research, Vol. 25, No. 5, 715-733, doi: 10.1142/S02175959 $\underline{08001973}$.

[10] Gupta, A., Singh, H., Aggarwal, A. (2011). Taguchi-fuzzy multi output optimization (MOO) in high speed CNC turning of AISI P-20 tool steel, Expert Systems with Applications, Vol. 38, No. 6, 6822-6828, doi: 10.1016/ j.eswa.2010.12.057.

[11] Dalman, H. (2016). An interactive fuzzy goal programming algorithm to solve decentralized bi-level multiobjec-

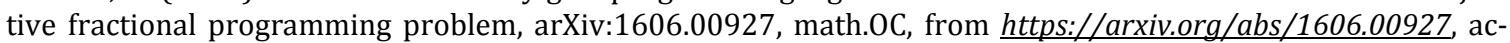
cessed August 7, 2020.

[12] Al-Refaie, A., Bani Domi, G., Abdullah, R. (2019). A fuzzy goal programming-regression approach to optimize process performance of multiple responses under uncertainty, International Journal of Management Science and En-gineering Management, Vol. 14, No. 1, 20-32, doi: 10.1080/17509653.2018.1467802.

[13] Ramakrishnan, R., Karunamoorthy, L. (2008). Modeling and multi-response optimization of Inconel 718 on machining of CNC WEDM process, Journal of Materials Processing Technology, Vol. 207, No. 1-3, 343-349, doi: 10.1016/i.jmatprotec.2008.06.040.

[14] Chen, C.-C., Tsao, C.-C., Lin, Y.-C., Hsu, C.-Y. (2010). Optimization of the sputtering process parameters of GZO films using the Grey-Taguchi method, Ceramics International, Vol. 36, No. 3, 979-988, doi: 10.1016/j.ceramint. 2009.11.019.

[15] Tahir, W., Jahanzaib, M., Raza, A. (2019). Effect of process parameters on cutting speed of wire EDM process in machining HSLA steel with cryogenic treated brass wire, Advances in Production Engineering \& Management, Vol. 14, No. 2, 143-152, doi: 10.14743/apem2019.2.317.

[16] Evran, S., Mutlu, B., Kurt, M. (2019). Cutting rate and surface characteristic analysis in CNC wire electrical discharge machining of aluminium bronze, Technical Gazette - Tehnički Vjesnik, Vol. 26, No. 5, 1228-1233, doi: $10.17559 / \mathrm{TV}-20180203185742$.

[17] Bhuyan, R.K., Routara, B.C., Parida, A.K. (2015). Using entropy weight, OEC and fuzzy logic for optimizing the parameters during EDM of Al-24\% SiCP MMC, Advances in Production Engineering \& Management, Vol. 10, No. 4, 217-227, doi: 10.14743/apem2015.4.204.

[18] Rao, P.S., Ramji, K., Satyanarayana, B. (2014). Experimental investigation and optimization of wire EDM parameters for surface roughness, MRR and white layer in machining of aluminium alloy, Procedia Materials Science, Vol. 5, 2197-2206, doi: 10.1016/j.mspro.2014.07.426. 


\title{
Optimizacija zmogljivosti procesa z različnimi peterokotnimi mehkimi odzivi: Študiji primera postopkov obdelave z žično elektroerozijo in postopkom naprševanja
}

\author{
Al-Refaie, A. ${ }^{\text {a }}$, Lepkova, N. ${ }^{\text {b, }}{ }^{*}$, Abbasi, G. ${ }^{\text {a }}$, Bani Domi, G. ${ }^{\mathrm{a}}$ \\ aUniversity of Jordan, Department of Industrial Engineering, Jordan \\ bVilnius Gediminas Technical University, Department of Construction Management and Real Estate, Lithuania
}

\section{POVZETEK}

Ta raziskava je razvila matematične modele za optimizacijo zmogljivosti procesa z različnimi peterokotnimi mehkimi odzivi. Sprva je bil vsak odziv predstavljen s funkcijo članstva $\mathrm{v}$ peterokotniku. Nato je bila za vsako ponovitev odziva pridobljena kombinacija optimalnih ravni dejavnikov. Te optimalne kombinacije so bile nato uporabljene za izdelavo peterokotnih regresijskih modelov za vsak odziv. Oblikovan in rešen je bil model peterokotne mehke optimizacije, s čimer se določi kombinacija optimalnih ravni dejavnikov pri vsakem elementu mehkega števila odziva peterokotnika. Za ponazoritev sta bili predloženi dve resnični študiji primera, in sicer obdelava z žično elektroerozijo in postopek naprševanja. Optimalni rezultati obeh študij so pokazali, da je predlagani model učinkovito optimiziral delovanje obeh procesov in kljub negotovosti zagotovil izboljšanje številnih kazalnikov kakovosti. Predlagani postopek lahko izboljša znanje procesnega inženirja o vplivih negotovosti na uspešnost procesa/izdelka in pomaga izvajalcem pri odločanju o ustreznih nastavitvah ravni dejavnikov za izboljšanje učinkovitosti postopkov obdelave $\mathrm{z}$ žično elektroerozijo in naprševanja.

(c) 2020 CPE, University of Maribor. All rights reserved.

\section{PODATKI O ČLANKU}

Ključne besede:

Modeliranje in optimizacija; Mehko programiranje ciljev; Peterokotno regresijsko modeliranje;

Peterokotna mehka števila;

Žična elektroerozija (WEDM);

Hrapavost površine (SR);

Stopnja odstranjevanja materiala

(MRR);

Postopek naprševanja;

$\mathrm{ZnO}$, dopiran z galijem (GZO)

*Kontaktna oseba:

natalija.lepkova@vgtu.lt

(Lepkova, N.)

Zgodovina članka:

Prejet 7. avgusta 2020

Popravljen 16. oktobra 2020

Sprejet 19. oktobra 2020 